\title{
On the calculation of offshore wind turbine load spectra for fatigue design
}

\author{
Rui Teixeira ${ }^{1}$, Maria Nogal $^{1}$, and Alan O’Connor ${ }^{1}$ \\ ${ }^{1}$ Trinity College Dublin, College Green, Dublin \\ rteixeiratcd.ie
}

\begin{abstract}
The present work researches on the definition of the load spectra used for offshore wind turbine low SN slope materials' fatigue design.

Uncertainty in the sample sized used to scale fatigue life is analyzed for the tower component. Damage density is investigated for different environmental conditions in order to understand the importance of the different regimes of operation. Damage density is identified to be a heterogeneous function of the loading environmental conditions. In some cases, even for low SN slope materials, most of the damage occurs due to high load ranges. To study on the influence of this heterogeneity, different statistical tail fits are used to compare the influence of accurately defining the tail region on a reference design time $(T)$.

Results show that OWT fatigue is highly dependent on the $t$ shorter that $T$ time used to approximate $T$. This is mainly related to the fact that fatigue design depends not only on scaling stress ranges, but also cycle counts. Effort on the design phase should be applied in the definition of the uncertainty of the load spectra due to the limitation imposed by using low sample sizes to cover the extensive joint distribution of environmental parameters.
\end{abstract}

Keywords: Offshore wind, Fatigue design, Miner's rule, 5MW Baseline OWT.

\section{$1 \quad$ Introduction}

Structural fatigue on OWTs is mainly divided in two major problems. For materials with high SN slopes (here considered as materials with SN slope $m>10$ ) the problem of fatigue approaches a problem of extrapolation, where the high loads contribute for almost all the fatigue damage density ( $\%$ of Miner's fatigue contribution). Whereas for low slope materials (here considered as materials with SN slope $m<6$ ), fatigue damage density is spread over different loading ranges.

Wide research on fatigue analysis of OWT has focused on the definition of the extrapolation techniques for composite materials [1-3]. For low SN slope materials, such as steels, knowledge on fatigue design techniques were transferred from the one already existing on offshore engineering. In particular, [3] reported that no additional accuracy was attained by spending effort on definition of the load spectra tail for low $m$ materials. To note that the authors use a sample size per environmental loading condition larger than the recommended by the current design standards. 
The current paper proposes to research on how the sample size influences fatigue calculations. Additionally, as the tail region is identified to be influent in some operational points, the trade-off of spending time on defining it is studied. To fulfill this goal, Section 2 presents the fatigue design calculation process, Section 3 presents the results on its uncertainty for 25 points of operation, and Section 4 presents the main conclusions.

\section{$2 \quad$ Fatigue design methodology}

Design of offshore wind turbine (OWT) towers to structural fatigue is an effort demanding procedure. The current design practices recommend the definition of load distributions, extrapolation of loads and cycles when required, to be used with the widely known linear damage summation rule, Equation $1[4,5]$.

$$
D_{T}=\sum_{i=1}^{S_{k}} \frac{n_{E}\left(S_{i}\right)}{n_{S N}\left(S_{i}\right)}
$$

$n_{E}\left(S_{i}\right)$ is the number of cycles at a certain stress range $S_{i}$, which is compared with the admissible number of cycles $n_{S N}\left(S_{i}\right)$ at that same stress range. $n_{S N}\left(S_{i}\right)$ is calculated based on the a material specified SN curve and $k$ is the total number of stress ranges.

The calculation of $n_{E}\left(S_{i}\right)$ frequently involves using a cycle counting technique, being the most common the rainflow cycle counting technique. Cycle counting techniques allow for the definition of a stress or load range spectra. Furthermore, as it is not feasible to perform the calculations replicating all the operational states an OWT may experience during its $T$ lifetime, calculations are performed for a limited number of environmental conditions and then scaled up for $T$.

The definition of the load spectra enables the assessment of $D_{T}$ using an integration technique, Equation 2.

$$
D_{T}=\int_{\Theta} \int_{S} \frac{f(\Theta) f(S \mid \Theta)}{n_{S N}(S)} d S d \Theta
$$

being $f(\Theta)$ the distribution function of operational conditions that load the OWT. This distribution is integrated along with the distribution of stress ranges $f(S \mid \Theta)$. $f(S \mid \Theta)$ is estimated from the stresses and cycles obtained at $\Theta$, being conditional on it. As multiple $\Theta$ load the OWT, the calculation procedure is expensive even when $f(S \mid \Theta)$ is defined with small sample sizes.

\section{$2.1 \quad$ OWT model}

The analysis presented uses the NREL 5MW baseline turbine installed on a monopile [6]. As a non-linear coupled code, it replicates the randomness of the OWT tower operational loading. Its non-linear behavior is of relevance to replicate non-linear loading behavior that may be of relevance for structural fatigue design. This is particularly important for high SN slope materials. However, it may be also of relevance for low SN slope materials. 
$[4,5]$ recommend the usage of at least six distinct seeds in order to define the design loads of OWTs to operational fatigue. Lower frequency loading effects have negligible influence on fatigue life [7]. Damage that occurs within this reference time $t$ of 600s much shorter than $T$ is identified as $D_{t}$.

\section{Analysis of fatigue load spectra uncertainty}

Short term fatigue follows a lognormal distribution. As many repetitions occur within the $\mathrm{T}$ period, cumulated fatigue converges to a single value that is related to the mean of $f(S \mid \Theta)$. Most of the times defining the mean value of $f(S \mid \Theta)$ maintaining practicable computational effort results in uncertainty in the estimation.

The approximation of the fatigue design calculations to a problem of mean value was considered before for wind turbines in [2]. Figure 1 presents the uncertainty in the calculation of the mean using 8 repetitions $\left(n_{r}=8\right)$ of six different seeds for the cumulative damage calculation. The mean wind speed $(U)$ and the turbulence intensity $(I)$ were selected to compute the results as these are expected to be the most influential variables in terms of fatigue of the tower [8].

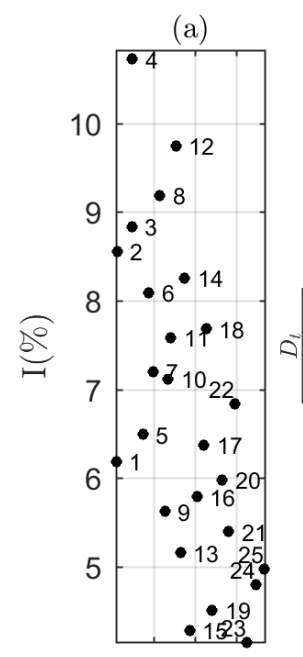

101520

$\mathrm{U}(\mathrm{m} / \mathrm{s})$
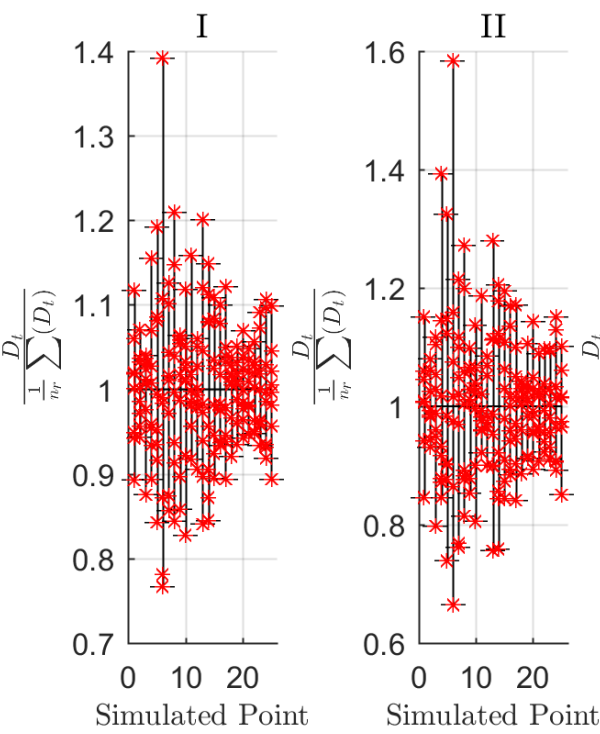

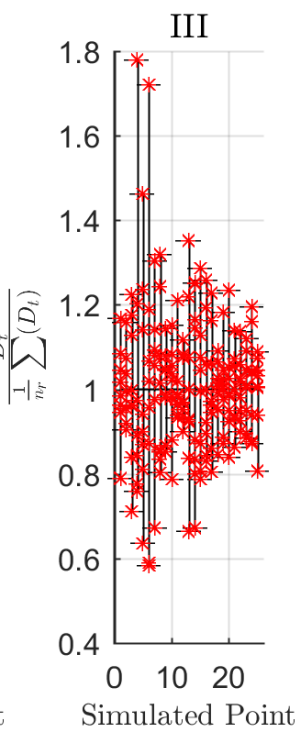

Simulated Point

- Simulated Points $\quad * \quad D_{t}-\frac{D_{t}}{\frac{1}{n_{r}} \sum D_{t}} \mp \max \Delta \frac{D_{t}}{\frac{1}{n_{r}} \sum D_{t}}$

Figure 1 - Uncertainty in the cumulated damage $\left(D_{t}\right)$ resulting of 6 seeded simulations of $600 s$ for $n_{r}=8$. (a) - Simulated Points. (I-III) $-\mathrm{SN} \mathrm{m}=3,4,5$ respectively.

It can be seen in Figure 1 that the variability of the mean within the $n_{r}$ calculations can be relatively high when using 6 seeds. In particular for high values of $I$ this variability is more prominent. To note that offshore $I$ is correlated negatively with $U$, therefore, the most turbulent operational states are expected to occur at less damaging 
winds. Nevertheless, even for the $\Theta$ where the variability of $D_{t}$ is relatively smaller (near the cut-out speed of $25 \mathrm{~m} / \mathrm{s}$ ) deviations of $\pm 10 \%$ are frequently identified. The calculations at $D_{t}$ are used to scale up $D_{T}$ depending on the occurrences of $f(\Theta)$. Thus, eventual errors in the estimation of the $D_{t}$ will be propagated to $D_{T}$. These errors are expected to average over 0 , however, if an inefficient description of $f(\Theta)$ occurs, or important fatigue damaging states are underestimated, relevant errors may be propagated to the design value $D_{T}$.

As the value of $m$ increases, the uncertainty in the cumulated damage increases for the same initial sample. This is connected to the influence of the higher quantiles of stress range. Figure 2 presents the damage density of the lower $95 \%$ quantile of the stress ranges $\left(D_{t: Q 95}\right)$ compared with the respective cumulative $D_{t}$ using 6 seeds, and its variability for $n_{r}$.
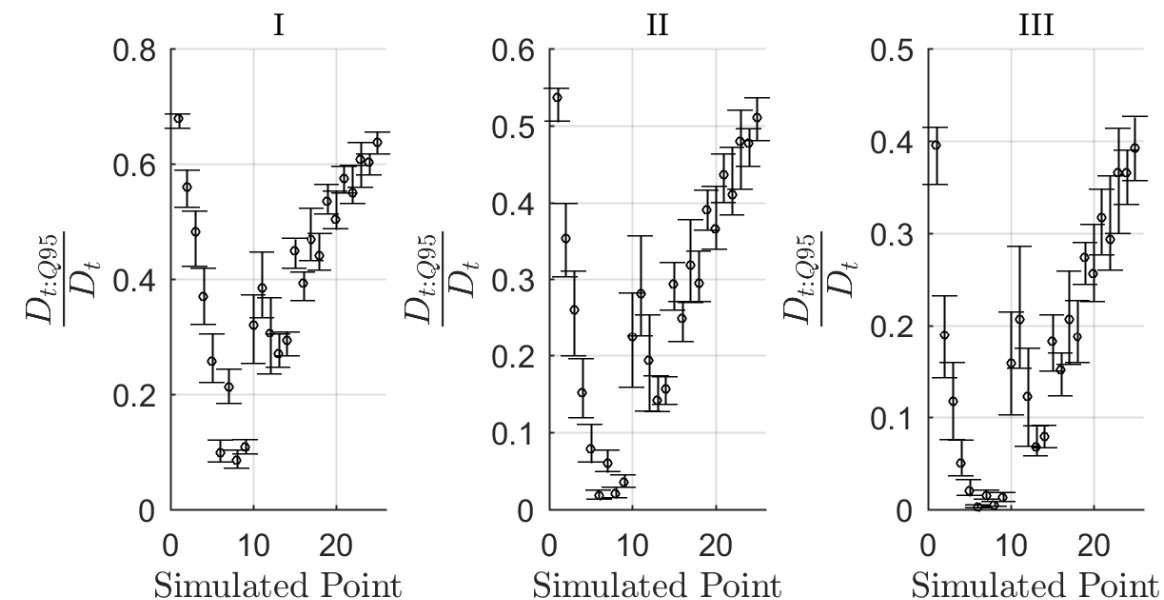

$$
\text { ○ } \frac{1}{n_{r}} \sum\left(\frac{D_{t: Q 95}}{D_{t}}\right) \quad \max \Delta\left(\frac{D_{t: Q 95}}{D_{t}}\right)
$$

Figure 2 - Density of $D_{t}$ for the lower $95 \%$ stress ranges and respective variation within $n_{r}$ repetitions. (I-III) $-\mathrm{SN} \mathrm{m}=3,4,5$ respectively.

As $m$ increases, influence of larger stress ranges increase. The variation of this percentage within the different simulated points is relatively low, which indicates that it is characteristic of each $\Theta$. Damage density in the tail can be as high as $95 \%$. It is then of interest to infer if design effort on the tail region should be spent for all the operational states, or even some, in order to have more accurate predictions of the $D_{T}$.

Characterization of the load spectra tail can be performed through extreme value theory or data truncation, being the second well accepted for wind engineering [3]. The challenge of extrapolation with truncation of data has been widely discussed before $[3,9,10]$. It is commonly assumed that low $\mathrm{SN}$ materials do not require the usage of extrapolation techniques to fit the tail distribution. Results from scaling the load spectra, considering and not considering extrapolation, from $t$ with 6 seeded simulations to $T$ equal to 50 simulations are discussed for low SN materials in the 
following section. The question to answer is if there is a significant advantage in terms of accuracy from adding the extra effort on an accurate load spectra tail estimation.

\subsection{Scaled results of $D$ from $t=3600 s$ to $T=60000 s$}

Comparative results from approximating the 100 points $D_{T}$ using $t$ of 6 seeded simulations at the same $\Theta$ state are presented in Table 1. The Peak-Over-Threshold (POT) methodology is applied and loading quantiles of $95 \%$ and $99 \%$ as threshold $(u)$ value. Extensive considerations about the POT procedure are given in [10]. The choice of these quantiles, previously identified as inappropriate [9], is due the systematic character required for the procedure.

Table 1 - Estimation of $D$ for $T=30000 s$ using $t=3600$ s. $D_{G P}, D_{W}$ and $D_{E}$ are relative to the tail fit with the Generalised Pareto, the Weibull and the Exponential distributions.

\begin{tabular}{|c|c|c|c|c|c|c|c|c|c|c|c|c|c|c|}
\hline \multirow[b]{3}{*}{$\theta$} & \multicolumn{7}{|c|}{$m=3$} & \multicolumn{7}{|c|}{$m=5$} \\
\hline & \multirow{2}{*}{$\frac{\times T / t}{D}$} & \multicolumn{3}{|c|}{$u=Q 95$} & \multicolumn{3}{|c|}{$u=Q 99$} & \multirow{2}{*}{$\frac{\times T / t}{D}$} & \multicolumn{3}{|c|}{$u=Q 95$} & \multicolumn{3}{|c|}{$u=Q 99$} \\
\hline & & $D_{G P}$ & $D_{W}$ & $D_{E}$ & $D_{G P}$ & $D_{W}$ & $D_{E}$ & & $D_{G P}$ & $D_{W}$ & $D_{E}$ & $D_{G P}$ & $D_{W}$ & $D_{E}$ \\
\hline 1 & 0.94 & 1.04 & 1.02 & 1.05 & 0.95 & 0.95 & 0.95 & 0.92 & 1.39 & 1.20 & 1.45 & 0.96 & 0.97 & 0.97 \\
\hline 2 & 0.86 & 1.30 & 1.45 & 1.12 & 0.88 & 0.88 & 0.89 & 0.86 & 3.96 & 6.46 & 2.06 & 0.92 & 0.94 & 1.00 \\
\hline 3 & 1.06 & 1.68 & 2.33 & 1.36 & 1.11 & 1.13 & 1.13 & 1.29 & 5.90 & 18.86 & 2.63 & 1.55 & 1.59 & 1.72 \\
\hline 4 & 0.95 & 1.63 & 1.43 & 1.71 & 1.02 & 1.07 & 1.05 & 0.93 & 3.70 & 2.33 & 4.35 & 1.20 & 1.34 & 1.48 \\
\hline 5 & 1.12 & 2.34 & 2.07 & 2.09 & 1.19 & 1.19 & 1.28 & 1.45 & 8.18 & 5.88 & 6.05 & 1.70 & 1.67 & 2.51 \\
\hline 6 & 1.00 & 1.99 & 2.87 & 1.40 & 1.13 & 1.18 & 1.22 & 1.12 & 5.19 & 13.77 & 2.18 & 1.48 & 1.59 & 2.14 \\
\hline 7 & 0.95 & 1.59 & 1.28 & 1.62 & 1.00 & 1.01 & 1.05 & 0.76 & 2.96 & 1.41 & 3.16 & 0.89 & 0.90 & 1.15 \\
\hline 8 & 1.20 & 1.86 & 1.79 & 3.08 & 1.26 & 1.28 & 1.26 & 1.25 & 3.28 & 2.51 & 12.61 & 1.39 & 1.43 & 1.41 \\
\hline 9 & 0.79 & 0.99 & 0.97 & 1.36 & 0.80 & 0.81 & 0.80 & 0.65 & 1.03 & 0.97 & 2.90 & 0.68 & 0.69 & 0.67 \\
\hline 10 & 0.88 & 1.00 & 0.97 & 1.03 & 0.89 & 0.89 & 0.89 & 0.84 & 1.17 & 1.03 & 1.34 & 0.86 & 0.87 & 0.86 \\
\hline 11 & 0.83 & 0.93 & 0.91 & 0.97 & 0.84 & 0.84 & 0.84 & 0.78 & 1.02 & 0.93 & 1.24 & 0.79 & 0.79 & 0.79 \\
\hline 12 & 0.85 & 1.00 & 0.98 & 1.20 & 0.86 & 0.86 & 0.86 & 0.79 & 1.13 & 1.08 & 2.24 & 0.80 & 0.80 & 0.80 \\
\hline 13 & 0.91 & 1.12 & 1.10 & 1.26 & 0.92 & 0.92 & 0.92 & 0.76 & 1.32 & 1.18 & 2.07 & 0.78 & 0.79 & 0.82 \\
\hline 14 & 0.98 & 1.25 & 1.20 & 1.41 & 1.00 & 0.99 & 1.00 & 0.97 & 1.76 & 1.46 & 2.80 & 1.01 & 1.00 & 1.05 \\
\hline 15 & 1.06 & 1.20 & 1.17 & 1.25 & 1.07 & 1.08 & 1.07 & 1.02 & 1.47 & 1.33 & 1.77 & 1.06 & 1.08 & 1.07 \\
\hline 16 & 0.96 & 1.18 & 1.13 & 1.24 & 0.99 & 1.00 & 1.00 & 0.98 & 1.79 & 1.42 & 2.24 & 1.05 & 1.07 & 1.07 \\
\hline 17 & 0.97 & 1.17 & 1.11 & 1.16 & 0.99 & 0.99 & 0.99 & 0.89 & 1.65 & 1.29 & 1.60 & 0.94 & 0.94 & 0.93 \\
\hline 18 & 1.06 & 1.37 & 1.32 & 1.56 & 1.10 & 1.07 & 1.13 & 1.29 & 2.55 & 2.15 & 4.30 & 1.45 & 1.33 & 1.77 \\
\hline 19 & 0.93 & 1.04 & 1.04 & 1.11 & 0.94 & 0.94 & 0.94 & 0.90 & 1.30 & 1.22 & 1.78 & 0.92 & 0.92 & 0.92 \\
\hline 20 & 0.95 & 1.06 & 1.05 & 1.14 & 0.96 & 0.96 & 0.96 & 0.95 & 1.30 & 1.25 & 1.84 & 0.97 & 0.98 & 0.99 \\
\hline 21 & 0.91 & 1.03 & 0.98 & 1.01 & 0.92 & 0.92 & 0.92 & 0.86 & 1.44 & 1.09 & 1.28 & 0.89 & 0.89 & 0.90 \\
\hline 22 & 1.03 & 1.15 & 1.13 & 1.17 & 1.04 & 1.05 & 1.04 & 1.01 & 1.49 & 1.33 & 1.64 & 1.06 & 1.09 & 1.07 \\
\hline 23 & 1.05 & 1.12 & 1.11 & 1.15 & 1.05 & 1.05 & 1.06 & 1.08 & 1.39 & 1.28 & 1.55 & 1.11 & 1.09 & 1.11 \\
\hline 24 & 0.98 & 1.06 & 1.04 & 1.07 & 0.99 & 0.99 & 0.99 & 0.99 & 1.32 & 1.16 & 1.39 & 1.01 & 1.01 & 1.01 \\
\hline 25 & 1.02 & 1.08 & 1.07 & 1.11 & 1.02 & 1.03 & 1.03 & 1.02 & 1.24 & 1.20 & 1.42 & 1.04 & 1.06 & 1.05 \\
\hline
\end{tabular}

The $u$ value of $95 \%$ can significantly overestimate the approximation to the tail, $\Theta=5$ ,8. The challenge of extrapolation for OWT is that of accurately predicting, not only the tail loading, but also the number of cycles. As a result, the confidence in the extrapolation is bounded to the number of initial samples applied. In the case of dealing with the tail region, six was proven to not be adequate by [3]. Up to 30 simulations the mean number of loading occurrences above the $99 \%$ quantile is highly variable. In most cases, no significant improvements occur in accuracy of the extra effort of studying the tail spectra region for the tower component. In the cases 9-12, where the simple scale up of the load spectra for $\frac{T}{t}$ is non-conservative, the usage of the POT with $95 \%$ quantile $u$ improves the estimation at $T$. Nonetheless, due to the complexity of analyzing with the POT, and variability in the accuracy, it may be more interesting to direct the design effort to quantify the uncertainty in the initial $t$ used. Further works, also for high $m$ values, should discuss the approximation of the extrapolated 
stress ranges and cycles simultaneously. It may be of interest to study the definition of $u$ based not only on quantiles of loading but also, or jointly, on a minimum number of expected cycles in order to implement extrapolation.

\section{Conclusions}

The current paper investigated uncertainty in the definition of loading spectra for offshore wind turbine tower component fatigue calculations. Variability due to sample statistical significance, with reference to the design standards, was studied. Damage density for different environmental conditions was also analyzed jointly with the improvement in accuracy resulting from the application of different statistical approximations. This was justified due to the heterogeneity of the damage density for different environmental conditions.

Results showed that, if practicality limits the design effort, resources should be focused on assessing uncertainty in the load spectra due to the sample size used to define it. Usage of complex statistical techniques, such as extrapolation, in certain circumstances improves the design accuracy, however, on its own, does not contribute for a robust design procedure and the resulting accuracy is highly case-specific.

\section{Acknowledgements}

This project has received funding from the European Union Horizon 2020 research and innovation programme under the Marie Sklodowska-Curie grant agreement No. 642453.

\section{References}

1. Sutherland, H. J. On the fatigue analysis of wind turbines. Tech. rep., SandiaNational Labs., Albuquerque, NM (US); Sandia National Labs., Livermore, CA (US) (1999).

2. Lange, C. H. Probabilistic fatigue methodology and wind turbine reliability. Tech. rep., Sandia National Labs., Albuquerque, NM, United States (1996).

3. Moriarty, P. J., Holley, W., Butterfield, S. Extrapolation of extreme and fatigue loads using probabilistic methods., National Renewable Energy Lab., Golden, CO (US) (2004).

4. IEC. Wind turbines part 1: Design requirements. Technical Report 61400-1, International Electrotechnical Commission, Geneva, Switzerland (2005).

5. IEC. Wind turbines part 3: Design requirements for offshore wind turbines. Technical Report 61400-3, International Electrotechnical Commission, Geneva, Switzerland (2009).

6. Jonkman, J., Butterfield, S., Musial, W., Scott, G. Definition of a 5-mw reference wind turbine for offshore system development. National Renewable Energy Laboratory, Golden, CO, Technical Report No. NREL/TP-500-38060 (2009).

7. Veldkamp, HF. "Chances in wind energy: a probalistic approach to wind turbine fatigue design." (2006).

8. Teixeira, R., O'Connor, A., Nogal, M., Krishnan, N., \& Nichols, J. Analysis of the design of experiments of offshore wind turbine fatigue reliability design with Kriging surfaces. Procedia Structural Integrity, 5, 951-958 (2017).

9. Embrechts, P., Resnick, S., Samorodnitsky, G. Extreme value theory as a risk management tool. North American Actuarial Journal 3 (2), 30- 41 (1999).

10. Teixeira, R., Nogal, M., O'Connor, A. On the suitability of the generalized Pareto to model extreme waves. Journal of Hydraulic Research (2018): 1-16. 